\title{
Resonant Raman scattering in ZnO:Mn and ZnO:Mn:Al thin films grown by RF sputtering
}

\author{
M. F. Cerqueira ${ }^{1}$, M. I. Vasilevskiy ${ }^{1}$, F. Oliveira ${ }^{1}$, A. G. Rolo ${ }^{1}$, T. Viseu ${ }^{1}$, \\ J. Ayres de Campos ${ }^{1}$, E. Alves ${ }^{2}$, R. Correia ${ }^{3}$ \\ ${ }^{1}$ Centro de Física, Campus de Gualtar, Universidade do Minho, 4710-057 Braga, \\ Portugal \\ ${ }^{2}$ ITN, Ion Beam Laboratory, E. N. 10, 2686-953 Sacavém, Portugal \\ ${ }^{3}$ Departamento de Física e I3N, Universidade de Aveiro, 3710-193 Aveiro, \\ Portugal
}

Corresponding e-mail address: fcerqueira@fisica.uminho.pt

\begin{abstract}
Raman spectroscopy results obtained under visible (non-resonant) and UV (resonant) excitation for nanocrystalline $\mathrm{ZnO}, \mathrm{ZnO}: \mathrm{Mn}$ and $\mathrm{ZnO}: \mathrm{Mn}: \mathrm{Al}$ thin films grown by RF sputtering are presented and compared. The origin of the multiple longitudinal optical (LO) phonon Raman peaks, strongly enhanced under resonance conditions, and the effects of the dopants on them are discussed in the framework of the "cascade" model. It is suggested that the observed suppression of the higher order LO phonon lines in $\mathrm{ZnO}: \mathrm{Mn}: \mathrm{Al}$ is caused by the dissociation of excitons in the heavily $n$-type doped material. Based on the cascade model interpretation of the higher order Raman peaks in the resonant spectra, the LO phonon frequencies for wavevectors away from the $\Gamma$ point are evaluated and compared to previously published phonon dispersion curves.
\end{abstract}

Keywords: $\mathrm{ZnO}$, phonons, Raman spectroscopy, $\mathrm{Mn}$ and $\mathrm{Al}$ doping

PACS numbers: 78.30.Fs, 61.72.uj 


\section{Introduction}

Zinc oxide $(\mathrm{ZnO})$ is a direct band gap semiconductor (band gap energy $E_{g}=$ $3.37 \mathrm{eV}$ at room temperature), considered as a good candidate for optoelectronic applications in the near UV region and for transparent electronics [1]. Moreover, since the famous prediction [2] of room temperature ferromagnetism in Mn-doped $\mathrm{ZnO}$ ( $\mathrm{ZnO}: \mathrm{Mn})$, it has been considered as a potentially very interesting material for spintronics. Raman scattering spectroscopy is a powerful non-destructive analytic technique which can provide useful information on the structure, morphology and chemical composition of semiconductor materials as well as on the photon-electron and electron-phonon interactions occurring in these materials [3]. Several earlier Raman studies have been performed on $\mathrm{ZnO}$ single crystals [4-6], as well as on nanostructured thin films and nanowires [6-13], both doped and undoped. These studies have demonstrated that non-resonantly excited Raman spectra, in general, follow rather well the symmetry-dictated selection rules characteristic of wurtzite crystal structure [4, 5]. At the same time, they revealed a strong dependence of the spectral features upon the nature and concentration of impurities present in $\mathrm{ZnO}$ samples [8-13]. Some of these features can be associated with local impurity vibrational modes [14] while some may be due to structural changes or impurity-induced lattice defects [8], grain size or secondary phases present in doped $\mathrm{ZnO}$ materials [15]. However, generally speaking, the knowledge of the lattice dynamics of $\mathrm{ZnO}$ is still rather limited as compared to most of III-V and II-VI semiconductors.

As far as resonant Raman scattering (RRS) is concerned, the spectra are simpler in the sense that the number of observed independent modes is lower. In fact, several studies [6, 8, 9] revealed just numerous replicas of the LO phonon mode (up to 11 [8]), likewise it had been observed for CdS in the classical works $[16,17]$ or more recently for $\mathrm{GaN}$ [18]. The intensity and number of LO phonon lines observed in RRS spectra of $\mathrm{ZnO}$-based materials depend on the grain size, impurities, defects, and free carrier concentration [8]. In a previous work [19] we studied the first-order (non-resonant) Raman response of $\mathrm{ZnO}$ films doped with different impurities including Mn. Here we report on our RRS results obtained on low-temperature-grown films of pure $\mathrm{ZnO}, \mathrm{ZnO}: \mathrm{Mn}$ and $\mathrm{ZnO}: \mathrm{Mn}$ co-doped with $\mathrm{Al}$ ( $\mathrm{ZnO}: \mathrm{Mn}: \mathrm{Al})$. The aim of this work has been to get a further insight into the dopant's behavior in the $\mathrm{ZnO}$ lattice and their effect on the exciton-phonon interaction that mediates RRS. We will show that the obtained results can be understood within the "cascade model", first proposed in Ref. [20] and further developed in Refs. [21-23], and discuss the interpretation of the relative intensities of the different multi-LO-phonon replicas in the spectra.

\section{Experimental procedure}

$\mathrm{ZnO}$ thin films were grown on silica substrates in an Alcatel SCM 650 sputtering system using a mixture of $\mathrm{O}_{2}$ and Ar gases (relative oxygen fraction of 0.23 ), at a constant working pressure of $0.7 \mathrm{~Pa}$ and at a substrate temperature of $50^{\circ} \mathrm{C}$. The target consisted of a hyper-pure $(99.99 \%)$ metal zinc wafer spaced $60 \mathrm{~mm}$ away from the substrates. Radio frequency (13.56 MHz) reactive sputter deposition was carried out after the chamber had reached a base pressure of $5 \times 10^{-5} \mathrm{~Pa}$. Using this technique we produced pure $\mathrm{ZnO}, \mathrm{ZnO}: \mathrm{Mn}$ and $\mathrm{ZnO}: \mathrm{Mn}: \mathrm{Al}$ layers. For the doping we used pieces of $\mathrm{Mn}$ and $\mathrm{Al}$ placed above the $\mathrm{Zn}$ target outside the erosion area. The $\mathrm{Mn}$ and Al contents in the samples were determined using Rutherford Backscattering Spectrometry (RBS) and Particle Induced XRay Emission (PIXE). For the $\mathrm{ZnO}: \mathrm{Mn}$ sample studied in this work, the RBS and PIXE data analysis yielded the Mn concentration of 1 at. $\%$ and for the 
$\mathrm{ZnO}: \mathrm{Mn}: \mathrm{Al}$ one the $\mathrm{Mn}$ and $\mathrm{Al}$ contents were evaluated at 1.5 at.\% and 0.12at.\%, respectively. The RBS analysis also confirmed the uniformity of the dopant concentration profile across the film. X-ray diffraction spectra of all films reveal the presence of a single phase with the hexagonal wurtzite structure and preferential orientation of the $c$-axis along the growth direction. The crystalline domain size was estimated at $\approx 180 \AA$. The undoped $\mathrm{ZnO}$, $\mathrm{ZnO}: \mathrm{Mn}$ and $\mathrm{ZnO}: \mathrm{Mn}: \mathrm{Al}$ films are completely transparent in the visible and near IR range. The thickness $(\approx 600 \mathrm{~nm})$ and optical parameters were determined using the Minkov method [24]. We found that the band gap energy slightly increases with the Mn content as expected [25].

Room temperature (RT) non-resonant Raman spectra were measured on a JobinYvon T64000 spectrometer equipped with a liquid nitrogen cooled CCD detector, in a back-scattering geometry, using the 514.5 $\mathrm{nm}$ excitation line of an $\mathrm{Ar}+$ laser with an incident power of $4 \mathrm{~mW}$. RRS spectra were measured at RT using a Jobin-Yvon LabRaman HR spectrometer equipped with a multi-channel air cooled $\left(-70^{\circ} \mathrm{C}\right) \mathrm{CCD}$ detector, in the back-scattering geometry, using the $325 \mathrm{~nm}$ excitation line of a $\mathrm{He}-\mathrm{Cd}$ laser. The photon energy $(3.815 \mathrm{eV})$ is well above $E_{g}$ for all the films studied.

\section{Results and discussion}

Figure 1 shows non-resonant Raman spectra of the studied $\mathrm{ZnO}, \mathrm{ZnO}: \mathrm{Mn}$ and ZnO:Mn:Al samples. They are similar to those reported in our previous work [19]. Vertical lines in Fig. 1 indicate the positions of the phonon modes previously observed for $\mathrm{ZnO}$ and $\mathrm{ZnO}: \mathrm{Mn}$. Among them, there are those allowed by the symmetry selection rules characteristic of wurtzite structure crystals $[4,5]$, namely, the $E_{2}$-low and $E_{2}-$ high non-polar phonon modes, the $A_{1}$ and $E_{1}$ transverse optical (TO) phonons, and the $A_{1}$ longitudinal optical (LO) phonon $\left(574 \mathrm{~cm}^{-1}\right)$. The $E_{1}$ LO mode (584 $\mathrm{cm}^{-1}$ according to [4] $587-591 \mathrm{~cm}^{-1}$ according to $\left.[5,26]\right)$, strictly speaking, cannot be observed in a backscattering geometry as a dipole-allowed mode. Still, there are mechanisms that can make it Raman-active in doped [18] and nanostructured [8] wurtzite crystals and especially under resonant excitation [3].

The broad peak at about $330 \mathrm{~cm}^{-1}$, seen in the spectrum of pure $\mathrm{ZnO}$ has been attributed to the second-order Raman processes involving acoustic phonons. Its presence demonstrates a good quality of the sample which is probably deteriorated with doping. The characteristic $437 \mathrm{~cm}^{-1}$ peak, assigned to the non-polar $E_{2}$ optical phonon mode, is present for pure and Mn-doped $\mathrm{ZnO}$ thin films, with a similar shape. The spectrum of the $\mathrm{ZnO}: \mathrm{Mn}: \mathrm{Al}$ sample appears rather structure-less, which might be attributed to a change in the surface morphology caused by the in situ co-doping with Al. In the previous work [19] the Al doping was achieved by ion implantation and the corresponding Raman spectrum (Fig. $2 b$ in Ref. [19]) is rather similar to that of $\mathrm{ZnO}: \mathrm{Mn}$.

The presence of the intense Mn-related mode at $\approx 530 \mathrm{~cm}^{-1}$ is clear in all Mn-doped samples. In our sputtering-grown samples, this mode appears only when $\mathrm{Mn}$ is present and its intensity has been shown to increase with increasing the Mn concentration [19]. We believe that it is an indication that $\mathrm{Mn}$ substitutes $\mathrm{Zn}$ in the lattice sites and the 530 $\mathrm{cm}^{-1}$ mode is related to a local vibration involving $\mathrm{Mn}$ atom. However, similar impurity-related Raman modes at frequencies close to $530 \mathrm{~cm}^{-1}$ have been reported for $\mathrm{ZnO}$ materials not containing Mn [8, 27], so we cannot exclude that our $530 \mathrm{~cm}^{-1}$ mode can be related to the presence of $\mathrm{Mn}$ in the sample only indirectly. For instance, it might be associated with defects in the host lattice induced by the doping [8].

Let us turn to the resonant Raman scattering results obtained on the same samples, shown in Fig. 2. The energies of the incoming and inelastic-scattered (shifted up to $4000 \mathrm{~cm}^{-1}$ ) photons match real electronic states in the material. 


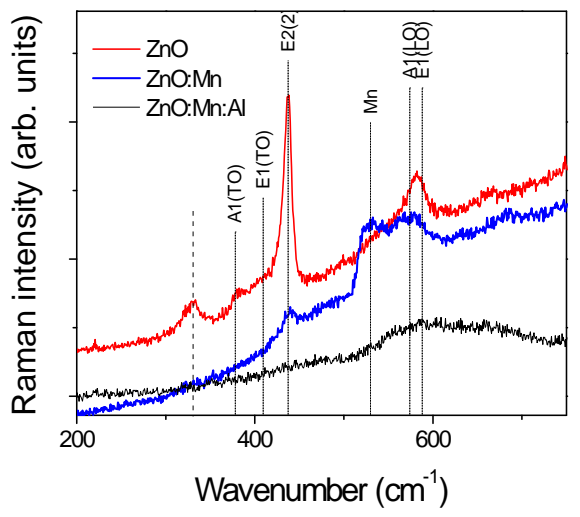

Figure 1: (colour online) Non-resonant Raman spectra of pure $\mathrm{ZnO}, \mathrm{ZnO}: \mathrm{Mn}$ and ZnO:Mn:Al samples. Vertical lines indicate the positions of the previously observed phonon modes.

According to several previous studies $[6,8$, 9], under resonant scattering conditions the Raman spectra of bulk $\mathrm{ZnO}$-based materials consist of only LO phonon lines and our spectra of Fig. 2 are also of this type (except for an extra mode marked "?", seen for the $\mathrm{ZnO}: \mathrm{Mn}: \mathrm{Al}$ sample*).

What are these LO phonons? The measured shift of $\approx 580 \mathrm{~cm}^{-1}$ is between the frequencies of the $A_{1}$ and $E_{1} \mathrm{LO}$ phonon modes at $\vec{k}=0$. As known [28], for a small (finite) wavevector making an angle $\vartheta$ with the $c$-axis of a uniaxial crystal, the LO phonon mode is a mixture of the Brillouin zone center $A_{1}$ and $E_{1}$ LO phonon modes, with the frequency given by $\omega_{L O}^{2}(\vartheta)=$ $\omega_{A 1}^{2} \cos ^{2} \vartheta+\omega_{E 1}^{2} \sin ^{2} \vartheta$. The observed RRS peak frequency corresponds to the maximum of $\left\langle\left(\omega^{2}-\omega_{L O}^{2}(\vartheta)\right)^{-1}\right\rangle$ where $\langle\ldots\rangle$ means an appropriate average over $\vartheta$. Indeed, the resonant enhancement of the (dipole-forbidden) LO scattering is associated with the Fröhlich electron-

* This mode seems to be related to the Al doping and is enhanced under resonance conditions. Its nature requires further investigation. phonon interaction mechanism and corresponds to the participation of LO phonons with small but finite wavenumbers [3]. As mentioned in the Introduction, the intensity and number of LO phonon lines observed in RRS spectra of different II-VI and III-V materials can depend on several factors but generally it is proportional to the strength of the exciton-phonon coupling that can be measured by the Huang-Rhys parameter (HRP). Based on the HRP value for $\mathrm{ZnO}$ [29], it has been predicted that the number of multiple LO phonon lines in bulk $\mathrm{ZnO}$ crystals should be higher than that of CdS with 9LO $(n=9)[16,17]$.

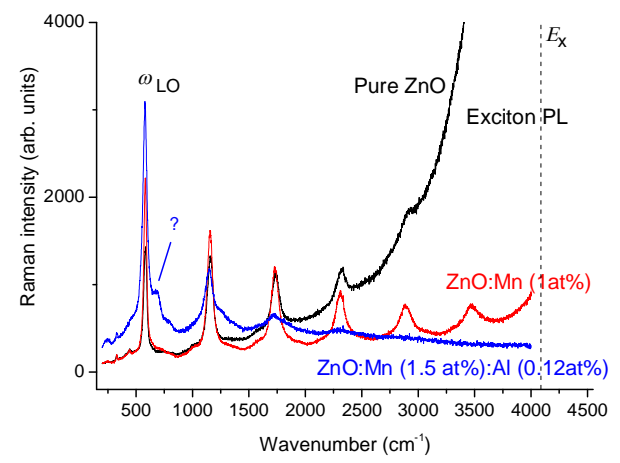

Figure 2: (colour online) Resonant Raman spectra of pure $\mathrm{ZnO}, \mathrm{ZnO}: \mathrm{Mn}$ and $\mathrm{ZnO}: \mathrm{Mn}: \mathrm{Al}$ films. The increase in the signal from pure $\mathrm{ZnO}$ at higher wavenumbers corresponds to excitonic photoluminescence (PL). The vertical line denoted $E_{x}$ shows the expected position of the center of this PL band.

However, in most of the previous studies a smaller number of the multi-LO-phonon lines has been observed, $n=4-8$ for pure $\mathrm{ZnO}[9,30]$ and $n=3-5$ for doped $\mathrm{ZnO}$ materials $[11,31]$. On the other hand, in a recent work [8] up to 11 replicas were resolved in the spectra of $\mathrm{ZnO}$ nanowires co-doped with two impurities.

In our data presented in Fig. 2, the number of the multi-phonon lines seen is also different for different samples, as is the full width half maximum value (FWHM). In 
order to quantify the intensity of the $n$-LO lines in the RRS spectra, we fitted them with a set of Lorentzians centered at the shifts equal to $n \hbar \omega_{L O}$, with adjusted FWHM and amplitude. For the pure $\mathrm{ZnO}$ sample, a Lorentzian contour representing the free exciton photoluminescence (PL) band (centered at the exciton energy, $3.31 \mathrm{eV}$ at RT [1], equivalent to the Raman shift of $4070 \mathrm{~cm}^{-1}$ with respect to the incident photon energy) was subtracted. The area of each $n$-LO Lorentzian, considered as the intensity of the corresponding multi-phonon peak, is plotted in Fig. 3.

As it can be seen from Fig. 3, already doping of $\mathrm{ZnO}$ with (electrically inactive) Mn reduces the intensity of the $n=2$ peak (relative to $n=1$ ). In principle, it could be explained by a (small) variation of the exciton-phonon coupling strength in the presence of $\mathrm{Mn}$ since Mn atoms deform the crystal lattice and, in principle, affect the properties of free excitons. However, we notice that the free exciton PL is suppressed by the Mn doping (it should be seen at a smaller Raman shift because of the increase of $E_{g}$ ). Still, we can observe at least six LO phonon replicas in the RRS spectrum. The extra presence of (electrically active) aluminum, comparing $\mathrm{ZnO}: \mathrm{Mn}$ and $\mathrm{ZnO}: \mathrm{Mn}: \mathrm{Al}$ samples, is expected to have more drastic effects on the exciton properties. In particular, high concentration of free carriers should destroy excitons because of the screening of the electron-hole interaction. Indeed, no exciton PL could be observed in this sample. Also, the number of multi-LO-phonon peaks and their intensity both decrease. Clearly, the relative intensities of the $n$-LO and $(n+1)-\mathrm{LO}$ are not determined by the strength of the excitonphonon coupling constant.

This puzzling situation was realized already in the 70-s and explained by the "cascade" model [20-23]. It has been shown that, so long as both the incident $\left(\hbar \Omega_{I}\right)$ and scattered $\left(\hbar \Omega_{S}\right)$ photon energies are larger than $E_{g}$, the multiple-LO-phonon "resonance Raman effect", in fact, is a sequence of (phonon-assisted) indirect processes of light absorption and emission by excitons [20].

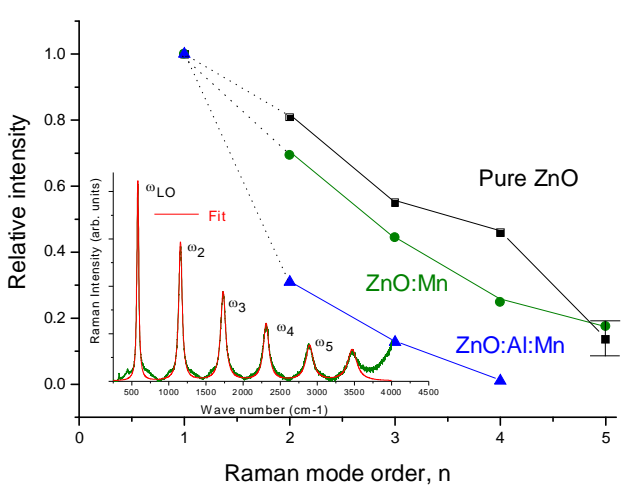

Figure 3: (colour online) Relative intensity of $n$-LO phonon lines in the RRS spectra of Fig. 2 (normalised to that of $n=1$ line for each sample). Lines are guides to the eye. Inset: RRS spectrum and corresponding fit for sample $\mathrm{ZnO}: \mathrm{Mn}$. Note that the mode frequencies $\omega_{i}(i=2,3, \ldots)$ are not exact multiples of $\omega_{L O}$.

According to the cascade model, the following sequence of elementary processes takes place: (i) the creation of an exciton via absorption of the incident photon $\left(\hbar \Omega_{I}\right)$, (ii) the (non-radiative) relaxation of this exciton into lower energetic states with successive emission of LO phonons, and (iii) the radiative recombination of the exciton with the emission of scattered phonons with energy $\hbar \Omega_{S}=\hbar \Omega_{I}-n \hbar \omega_{L O}(n>1)$, as schematically shown in Fig. 4. In our experiments $\hbar \Omega_{I}$ is approximately equal to $\left(E_{x}+7 \hbar \omega_{L O}\right)$ where $E_{x}=3.31 \mathrm{eV}$ is the ground state energy of free exciton. As illustrated in Fig. 4, the incident photon is absorbed into a virtual exciton state (marked by the cross). The "true" $n$-th order Raman process (left panel) involving the simultaneous emission of $n>1$ phonons is less efficient than the sequence of phononassisted absorption-emission (for $n=2$ ) or absorption-relaxation-emission (for $n>2$ ) 
processes, depicted in the right panel of Fig.4 [21].

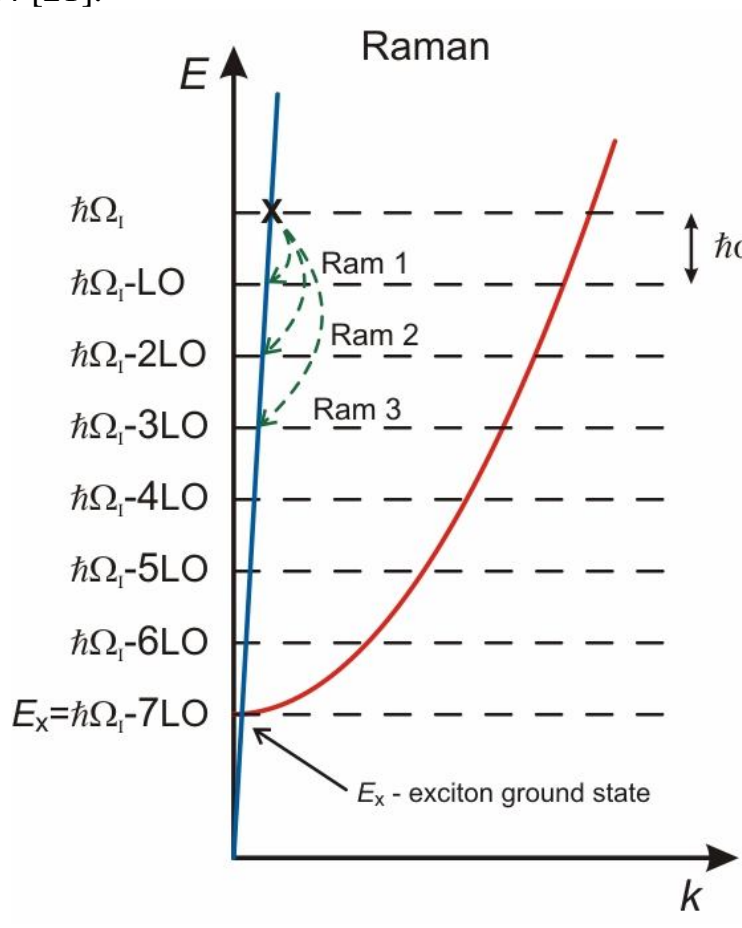

(1)

$\sigma_{2}=A \sigma_{A b s}\left(\hbar \Omega_{I}\right) \times$

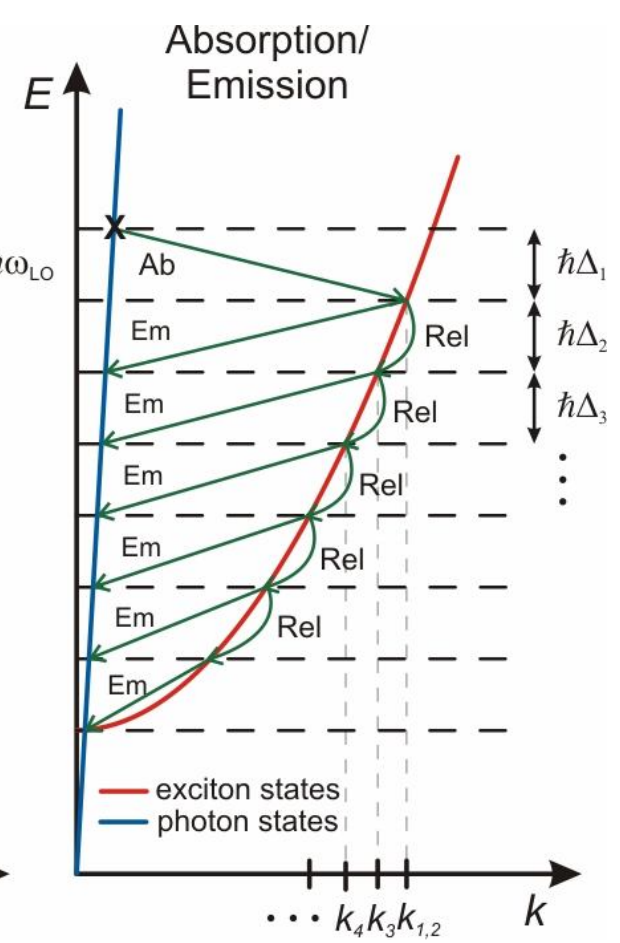

Figure 4: (colour online) Schematics of Raman (Ram1 - 3) and indirect absorption (Ab) /emission $(\mathrm{Em})$ processes leading to multi-phonon replicas in the emission spectra (adapted from Ref. 21). The abbreviation Rel means relaxation. Values of the wavevector indicated in the right panel, $k_{i}(i=1,2, \ldots)$ correspond to phonons interacting with the exciton in different states. Note that $\Delta_{i}(i=1,2, \ldots)$ shown in the right panel are not exactly equal to $\omega_{L O}$.

Therefore, according to the cascade model, only the first-order spectral peak corresponds to the "true Raman process" (denoted by Ram1 in Fig. 4) and its crosssection $\left(\sigma_{1}\right)$ is determined by the (Fröhlich) interaction between hot excitons and LO phonons with $\vec{k} \approx 0$.

The other peaks represent the exciton PL emission processes assisted by absorption and emission of phonons. In particular, the second-order process is physically different from the 1LO scattering, involves phonon absorption and emission (Ab+Em in Fig. 4) and its cross-section can be evaluated as [23]:

$$
\left[\frac{W_{E m}\left(\hbar \Omega_{I}-\hbar \omega_{L O}\right)}{W_{N r}\left(\hbar \Omega_{I}-\hbar \omega_{L O}\right)+W_{E m}\left(\hbar \Omega_{I}-\hbar \omega_{L O}\right)}\right]
$$

where the constant $A$ is of the order of unity, $\sigma_{A b s}(E)$ is the cross-section of the indirect photon absorption, $W_{E m}(E)$ is the probability of indirect annihilation of an exciton with kinetic energy $E$, accompanied by the emission of one LO phonon, and $W_{N r}$ is the probability of all non-radiative processes involving the exciton (relaxation and dissociation). Comparing $\sigma_{2} / \sigma_{1}$ for the three samples we can conclude that $W_{N r}$ for hot excitons must be much larger in the $\mathrm{ZnO}: \mathrm{Mn}: \mathrm{Al}$ sample, 
probably because of the high probability of the exciton dissociation in the presence of free carriers.

In analogy with Eq. (1), one can write for the cross-section of the third-order process:

(2) $\sigma_{3}=\sigma_{2}\left[\frac{W_{N r}\left(\hbar \Omega_{I}-\hbar \omega_{L O}\right)}{W_{E m}\left(\hbar \Omega_{I}-\hbar \omega_{L O}\right)}\right] \times$

$\left[\frac{W_{E m}\left(\hbar \Omega_{I}-2 \hbar \omega_{L O}\right)}{W_{N r}\left(\hbar \Omega_{I}-2 \hbar \omega_{L O}\right)+W_{E m}\left(\hbar \Omega_{I}-2 \hbar \omega_{L O}\right)}\right]$.

Similar to the relative intensity of the $2 \mathrm{LO}$ line, the $\sigma_{3} / \sigma_{2}$ ratio is also the lowest for the $\mathrm{ZnO}: \mathrm{Mn}: \mathrm{Al}$ sample, however, from Fig. 3 we can conclude that $\sigma_{3} / \sigma_{2}>\sigma_{2} / \sigma_{1}$ as it should be according to Eq. (2). For the sake of completeness, we note that this simple recurrent relation neglects (phononassisted) exciton transitions to higher energy states and becomes increasingly incorrect for larger $n$. If multi-phonon lines are observed for orders $n>n_{\text {max }}=$ $\left[\left(\hbar \Omega_{I}-E_{x}\right) / \hbar \omega_{L O}\right]$, as it is the case of some samples in Ref. 8, they cannot be described by the cascade model and should be regarded as truly Raman lines (left panel of Fig. 4).

From the fitting results presented in the inset of Fig 3, we verify that the separation between the consecutive Raman peaks is not exactly the same and not exactly equal to $\omega_{L O}$. It is naturally explained by the cascade model because higher order processes involve phonons with smaller wave vectors (see right-hand side Fig. 4). Therefore it opens the possibility to determine the dispersion relation for phonons taking part in the cascade process. The quantities $\Delta_{n}$ shown in Fig. 4 are the phonon frequencies and the corresponding values of the wavevector, $k_{n}$, can be calculated as explained below. For $n \geq 3$, we have $\Delta_{n}=\omega_{n}-\omega_{n-1}$ where $\omega_{n}$ are the shifts of the corresponding Raman peaks.
Table I: Frequencies of the phonons taking place in the cascade process

\begin{tabular}{|c|c|c|c|c|}
\hline$n$ & 1,2 & 3 & 4 & 5 \\
\hline$\Delta_{n}, \mathrm{~cm}^{-1}$ & 579.3 & 579.5 & 581.0 & 584.0 \\
\hline
\end{tabular}

Since $\Delta_{1,2}$ cannot be measured (the first peak has a different nature!), we take $\Delta_{1}=\Delta_{2}=\omega_{2} / 2$ and $k_{1}=k_{2}$, which is justified because the photon scanline in Fig. 4 is almost vertical. The phonon frequencies $\Delta_{n}$ obtained for the pure $\mathrm{ZnO}$ sample are given in Table I. Note that the 1-st Raman peak for the same sample is located at $\omega_{L O}=581.6 \mathrm{~cm}^{-1}$, i.e. $\omega_{L O}>\Delta_{1,2}, \Delta_{3}$ but at the same time $\omega_{L O}<\Delta_{5}$. It clearly indicates that the physical mechanisms responsible for the first and higher order peaks are different, in accordance with the cascade model.

The corresponding values of the wavevector can be calculated from the following relation,

(3) $E_{x}+\frac{\hbar^{2} k_{n}^{2}}{2 M}=\hbar\left(\Omega_{I}-\sum_{i=1}^{n-1} \Delta_{i}\right) \quad(n \geq 2)$,

where $M$ is the free exciton mass $\left(=0.9 m_{0}\right.$ for $\left.\mathrm{ZnO}[32]\right)$.

Phonon dispersion curves for bulk $\mathrm{ZnO}$ have recently been measured using inelastic neutron scattering (INS) [33]. In Fig. 5 we compare our results, $\Delta_{n}\left(k_{n}\right)$ with the data of Ref. 33. It should be pointed out that the INS measurements were performed at $10 \mathrm{~K}$ while our RRS data correspond to room temperature. Within the Grüneisen model, the relative shift of the phonon frequencies with temperature can be estimated as $\delta \omega / \omega=-\overline{\gamma \alpha} \delta T$, where $\bar{\gamma}$ and $\bar{\alpha}$ are the average Grüneisen parameter and average thermal expansion coefficient, respectively. With the data available from Ref. 34 we obtain for $\delta \omega_{L O}$ less than $0.5 \mathrm{~cm}^{-1}$, which is below our experimental precision. 


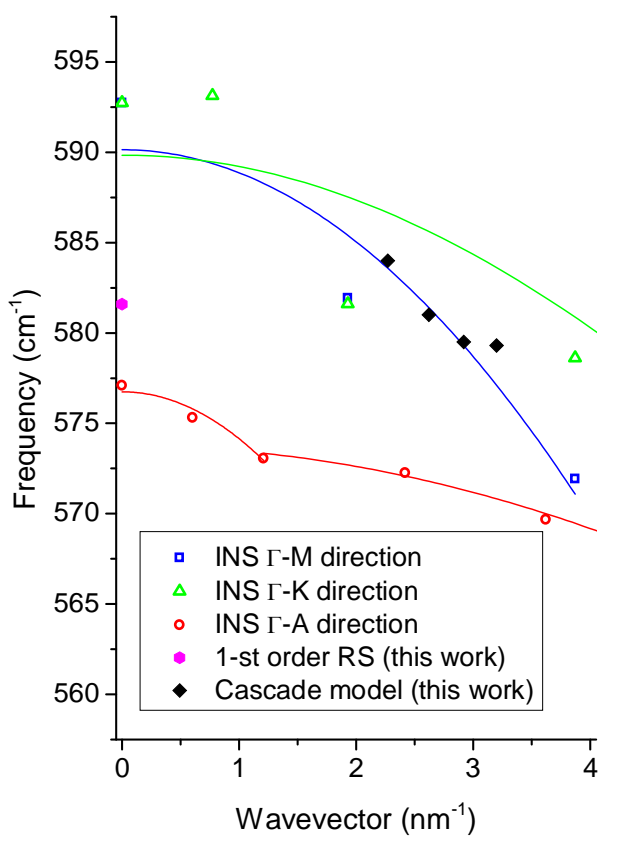

Figure 5: (colour online) $\mathrm{ZnO}$ LO phonon dispersion relations along $\Gamma-\mathrm{M}, \Gamma-\mathrm{K}$ and $\Gamma$ A directions in the Brillouin zone and our $\omega_{L O}$ and $\Delta_{n}\left(k_{n}\right)$ data (full symbols). Open symbols represent the INS data from [33] rescaled to the units used here and curves are parabolic fits to these data (guides to the eye).

From Fig. 5 we can see that our $\Delta_{n}\left(k_{n}\right)$ data fit quite well the LO phonon dispersion relation characteristic of the $x y$ plane $\left(E_{1}\right.$ type phonon modes). This is understandable because these polar phonons should be most strongly interacting with excitons and their contribution to the total density of states is larger compared to $A_{1}$ type phonon modes.

\section{Conclusions}

We observed the strong enhancement of the polar LO phonon mode in the 1-st order spectrum under resonant excitation, owing to the strong Fröhlich interaction between excitons and LO phonons. No enhancement of Mn-related Raman features in the spectra of $\mathrm{ZnO}: \mathrm{Mn}$ and $\mathrm{ZnO}: \mathrm{Mn}: \mathrm{Al}$ films was found, although a new feature, presumably related to Al doping, was detected in the RRS spectra. The intense multi-LO-phonon lines observed in the RRS spectra of all our $\mathrm{ZnO}$ films, which are also characteristic of many other II-VI and III-V materials, appear because of the indirect emission of light by hot excitons with appropriate energy, more efficient than the simultaneous emission of several phonons. This is confirmed, in particular, by the fact that the separation between the consecutive Raman peaks for $n \geq 2$ is different from the LO-phonon frequency measured by the position of the first peak. Moreover, such intense higher order peaks are not observed in the RRS spectra of 2-3 nm size crystallites (quantum dots) of the same materials [35]. Owing to the spatial quantization of the exciton energy spectrum in quantum dots, the cascade relaxation of hot excitons by emitting LO phonons is not possible there and the relaxation occurs via other processes involving the polaron formation [36]. For the samples studied in the present work, with the typical grain size of $20 \mathrm{~nm}$, the quantum confinement effect is not important. Using the cascade model [20-23], we evaluated the relation between the frequencies and wavevectors of phonons responsible for the higher order peaks in the RRS spectrum of pure $\mathrm{ZnO}$ film, which fits quite well the recently measured dispersion relation for $E_{1}$ type phonon modes in bulk $\mathrm{ZnO}$ [33]. The doping with $\mathrm{Mn}$ and $\mathrm{Al}$ influences the RRS spectra in different way and the characteristic features of this influence have also been understood in the framework of the cascade model. In particular, we suggest that the suppression of the higher order LO phonon lines, is caused by the dissociation of excitons in the heavily $n$-type doped $\mathrm{ZnO}$.

\section{Acknowledgments}

The authors wish to thank Prof. C. Trallero-Giner for insightful discussions. Financial support from the FCT through project PTDC/FIS/72843/2006 is acknowledged.

\section{References}

1. C. Klingshirn, J. Fallert, H. Zhou, J. Sartor, C. Thiele, F. Maler-Flaig, D. 
Schneider, and H. Kalt, Phys. Status Solidi B 247, 1424 (2010)

2. T. Dietl, H. Ohno, F. Matsukara, J. Gilbert and D. Ferrand, Science 287, 1019 (2000)

3. M. Cardona, In: Light Scattering in Solids II, edited by M. Cardona and G. Güntherodt, Topics in Applied Physics, Vol. 50 (Springer-Verlag, Berlin), Chap. 2 (1982).

4. J. M. Calleja and M. Cardona, Phys. Rev. B 16, 3753 (1977)

5. R. Cusco, E. Alarcon-Llado, J. Ibanez, L. Artus, J. Jimenez, B. Wang and M. J. Callahan, Phys. Rev. B 75, 165202 (2007)

6. V. V. Ursaki, I. M. Tiginyanu, V. V. Zalamai, E. V. Rusu, G. A. Emelchenko, V. M. Masalov and E. N. Samarov, Phys. Rev. B 70, 155204 (2004)

7. H. F. Liu, S. Tripathy, G. X. Hu, H. Gong, J. Appl. Phys. 105, 053507 (2009)

8. T. L. Phan, R. Vincent, D. Cherns, N. X. Nghia, and V. V. Ursaki, Nanotechnology 19, 475702 (2008).

9. B. Kumar, H. Gong, S. Y. Chow, S. Tripathy and Y. Hua, Appl. Phys. Lett. 89, 071922 (2006).

10. C. L. Du, Z. B. Gu, Y. M.Y ou, J. Kasim, T. Yu, Z. X. Shen, Z. H. Ni, Y. Ma, G. X. Cheng and Y. F. Chen, J. Appl. Phys. 103, 02352116 (2008)

11. R. B. Liu, A. L. Pan, H. M. Fan, F. F. Wang, Z. X. Shen, G. Z. Yang, S. S. Xie and B. S. Zou, J. Phys.: Condens. Matter 19, 136206 (2007)

12. C. Sudakar, J. S. Thakur, G. Lawes, R. Naik and V. M. Naik, Phys. Rev. B 75, 054423 (2007)

13. X. F. Wang, J. B. Xu, X. J. Yu, K. Xue, J. G. Yu and X. J. Zhao, Appl. Phys. Lett. 91, 031908 (2007)

14. P. Lakshmi and K. Ramachandran, Radiation Effects and Defects in Solids 161, 365 (2006)

15. K. Samanta, S. Dussan, R. S. Katiyar and P. Bhattacharya, Appl. Phys. Lett. 90, 261903 (2007)

16. R. C. C. Leite, J. C. Scott and T. C. Damen, Phys. Rev. Lett. 22, 780 (1969)
17. M. V. Klein and S. P. S. Porto, Phys. Rev. Lett. 22, 782 (1969)

18. W. H. Sun, S. J. Chua, L. S. Wang and X. H. Zhang, J. Appl. Phys. 91, 4917 (2002)

19. M. F. Cerqueira, A. G. Rolo, T. Viseu, J. Ayres de Campos, T. de Lacerda-Arôso, F. Oliveira, M. I. Vasilevskiy, and E. Alves, Phys. Status Solidi C 7, 2290 (2010).

20. R. M. Martin and C. M. Varma, Phys. Rev. Lett. 26, 1241 (1971)

21. E. Gross, S. Permogorov, Ya. Morozenko and B. Kharlamov, Phys. Status Solidi B 59, 551 (1973)

22. M. V. Klein, Phys. Rev. B 8, 919 (1973)

23. C. Trallero-Giner, I. G. Lang and S. T. Pavlov, Sov. Phys. Solid State 22, 718 (1980)

24. J. Ayres de Campos, T. Viseu, A. G. Rolo, N. P. Barradas, E. Alves, T. de Lacerda-Arôso, and M. F. Cerqueira, J. Nanosci. Nanotechnol. 10, 2620 (2010).

25. A. I. Savchuk, V. I. Fediv, S. A. Savchuk and A. Perrone, Superlattices and Microstructures 38, 421 (2005).

26. U. Özgür, Ya. I. Alivov, C. Liu, A. Teke, M. A. Reshchikov, S. Doğan, V. Avrutin, S.-J. Cho, and H. Morkoç, J. Appl. Phys. 98, 041301 (2005)

27. C. Bundesmann, N. Ashkenov, M. Schubert, D. Spemann, T. Butz, E. M. Kaidashev, M. Lorenz and M. Grundmann, Appl. Phys. Lett. 83, 1974 (2003).

28. W. Hayes, R. Loudon. Scattering of Light by Crystals; Wiley, New York, 1978.

29. J. F. Scott, Phys. Rev. B 2, 1209 (1970)

30. V. V. Zalamai, V. V. Ursaki, E. V. Rusu, P. Arabadji, I. M. Tiginyanu and L. Sirbu, Appl. Phys. Lett. 84, 5168 (2004)

31. J. D. Ye, K. W. Teoh, X. W. Sun, G. Q. Lo, D. L. Kwong, H. Zhao, S. L. Gu, R. Zhang, Y. D. Zheng, S. A. Oh, X. H. Zhang, and S. Tripathy, Appl. Phys. Lett. 91, 091901 (2007)

32. J. Fryar, E. McGlynn1, M. O. Henry, and J.-P. Mosnier. Nanotechnology 16, 2625 (2005) 
33. J. Serrano, F. J. Manjón, A. H. Romero, A. Ivanov, M. Cardona, R. Lauck, A. Bosak, and M. Krisch, Phys. Rev. B 81, 174304 (2010)

34. H. Iwananga, A. Kunishige, and S. Takeuchi, J. Mat. Sci. 35, 2451 (2000)

35. A. G. Rolo and M. I. Vasilevskiy, J. Raman Spectroscopy 38, 618 (2007)

36. T. Stauber and M. I. Vasilevskiy, Phys. Rev. B. 79, 113301 (2009) 


\section{Equations:}

Equation (1)

$\sigma_{2}=A \sigma_{A b s}\left(\hbar \Omega_{I}\right)\left[\frac{W_{E m}\left(\hbar \Omega_{I}-\hbar \omega_{L O}\right)}{W_{N r}\left(\hbar \Omega_{I}-\hbar \omega_{L O}\right)+W_{E m}\left(\hbar \Omega_{I}-\hbar \omega_{L O}\right)}\right]$

Equation (2)

$\sigma_{3}=\sigma_{2}\left[\frac{W_{N r}\left(\hbar \Omega_{I}-\hbar \omega_{L O}\right)}{W_{E m}\left(\hbar \Omega_{I}-\hbar \omega_{L O}\right)}\right]\left[\frac{W_{E m}\left(\hbar \Omega_{I}-2 \hbar \omega_{L O}\right)}{W_{N r}\left(\hbar \Omega_{I}-2 \hbar \omega_{L O}\right)+W_{E m}\left(\hbar \Omega_{I}-2 \hbar \omega_{L O}\right)}\right]$

Equation (3)

$E_{x}+\frac{\hbar^{2} k_{n}^{2}}{2 M}=\hbar\left(\Omega_{I}-\sum_{i=1}^{n-1} \Delta_{i}\right) ; \quad n \geq 2$

Table I. Frequencies of the phonons taking place in the cascade process

\begin{tabular}{|l|c|c|c|c|}
\hline$n$ & 1,2 & 3 & 4 & 5 \\
\hline$\Delta_{n}, \mathrm{~cm}^{-1}$ & 579.3 & 579.5 & 581.0 & 584.0 \\
\hline
\end{tabular}




\section{Figures captions:}

Figure 1: (colour online) Non-resonant Raman spectra of pure $\mathrm{ZnO}, \mathrm{ZnO}: \mathrm{Mn}$ and $\mathrm{ZnO}: \mathrm{Mn}: \mathrm{Al}$ samples. Vertical lines indicate the positions of the previously observed phonon modes.

Figure 2: (colour online) Resonant Raman spectra of pure $\mathrm{ZnO}, \mathrm{ZnO}: \mathrm{Mn}$ and $\mathrm{ZnO}: \mathrm{Mn}: \mathrm{Al}$ films. The increase in the signal from pure $\mathrm{ZnO}$ at higher wavenumbers corresponds to excitonic photoluminescence (PL). The vertical line denoted $E_{x}$ shows the expected position of the center of this PL band.

Figure 3: (colour online) Relative intensity of $n$-LO phonon lines in the RRS spectra of Fig. 2 (normalised to that of $n=1$ line for each sample). Lines are guides to the eye. Inset: RRS spectrum and corresponding fit for sample $\mathrm{ZnO}: \mathrm{Mn}$. Note that the mode frequencies $\omega_{i}(i=2,3, \ldots)$ are not exact multiples of $\omega_{L O}$.

Figure 4: (colour online) Schematics of Raman (Ram1-3) and indirect absorption $(\mathrm{Ab}) /$ emission $(\mathrm{Em})$ processes leading to multi-phonon replicas in the emission spectra (adapted from Ref. 21). The abbreviation Rel means relaxation. Values of the wavevector indicated in the right panel, $k_{i}(i=1,2, \ldots)$ correspond to phonons interacting with the exciton in different states. Note that $\Delta_{i}(i=1,2, \ldots)$ shown in the right panel are not exactly equal to $\omega_{L O}$.

Figure 5: (colour online) $\mathrm{ZnO}$ LO phonon dispersion relations along $\Gamma-\mathrm{M}, \Gamma-\mathrm{K}$ and $\Gamma-\mathrm{A}$ directions in the Brillouin zone and our $\omega_{L O}$ and $\Delta_{n}\left(k_{n}\right)$ data (full symbols). Open symbols represent the INS data from [33] rescaled to the units used here and curves are parabolic fits to these data (guides to the eye). 


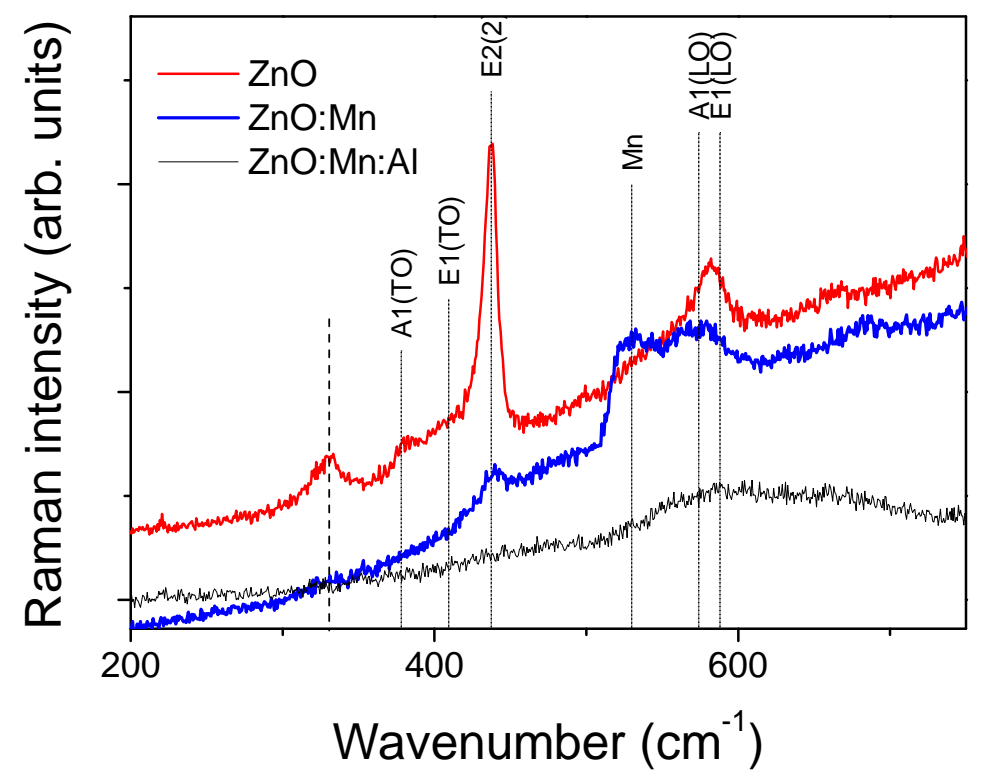

Figure 1 : 


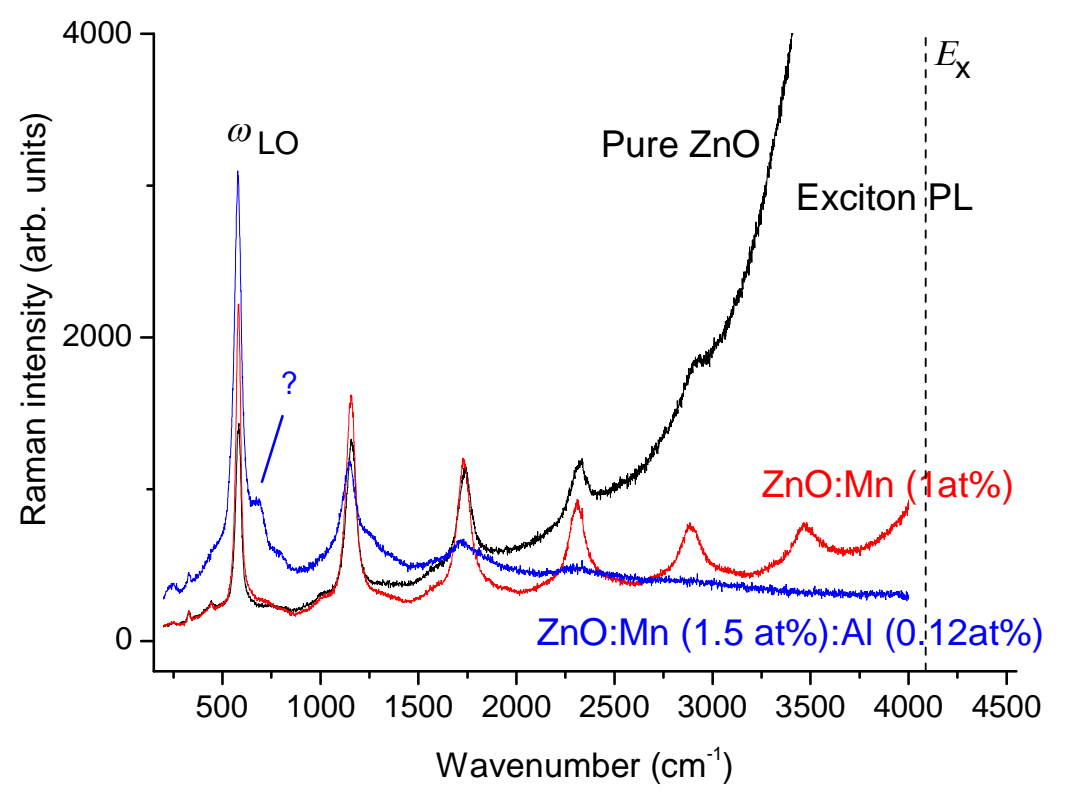

Figure 2 : 


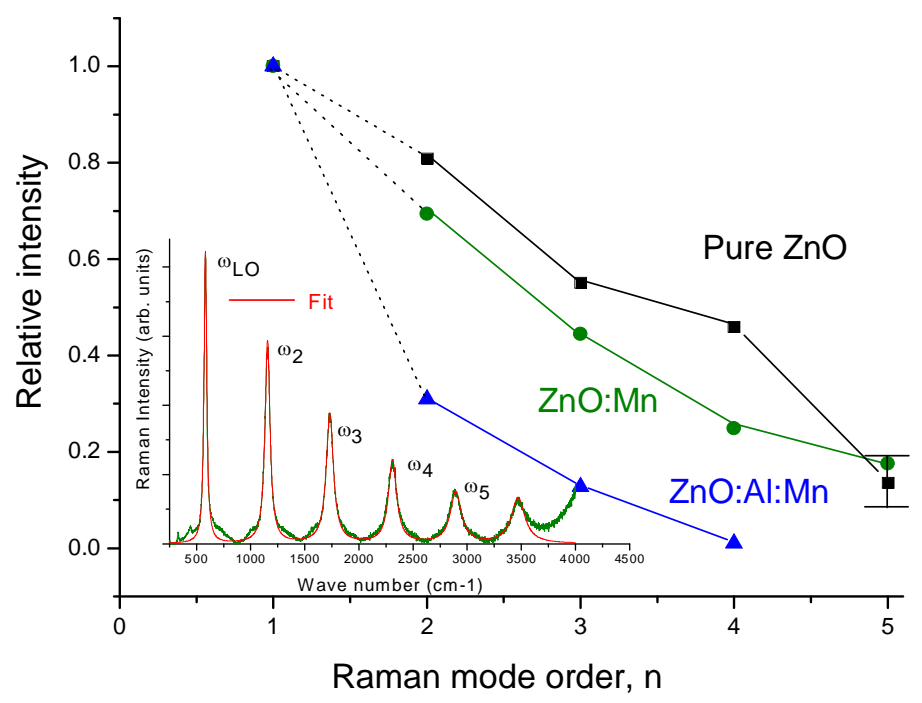

Figure 3 :

Create PDF files without this message by purchasing novaPDF printer (http://www.novapdf.com) 


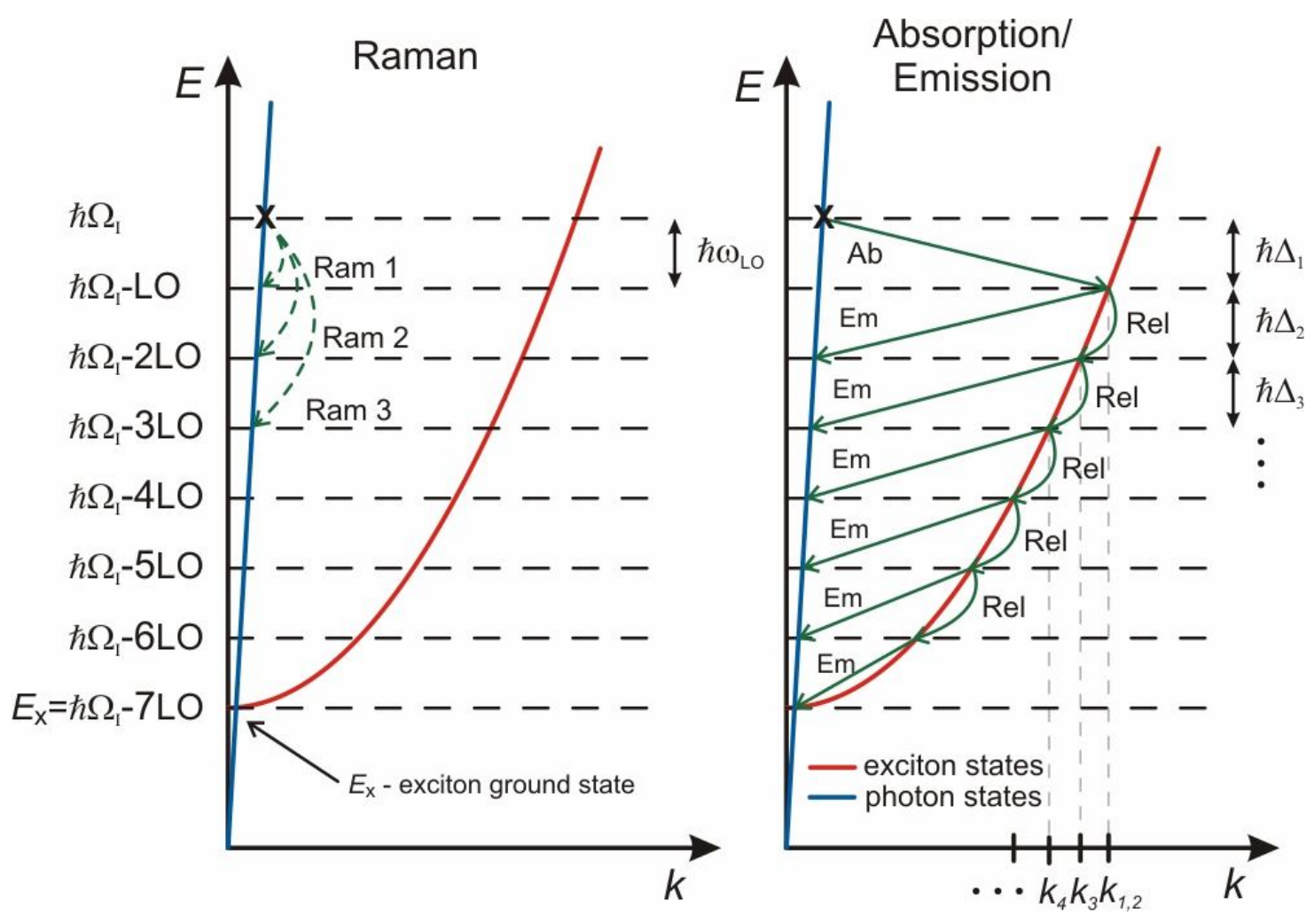

Figure 4 : 


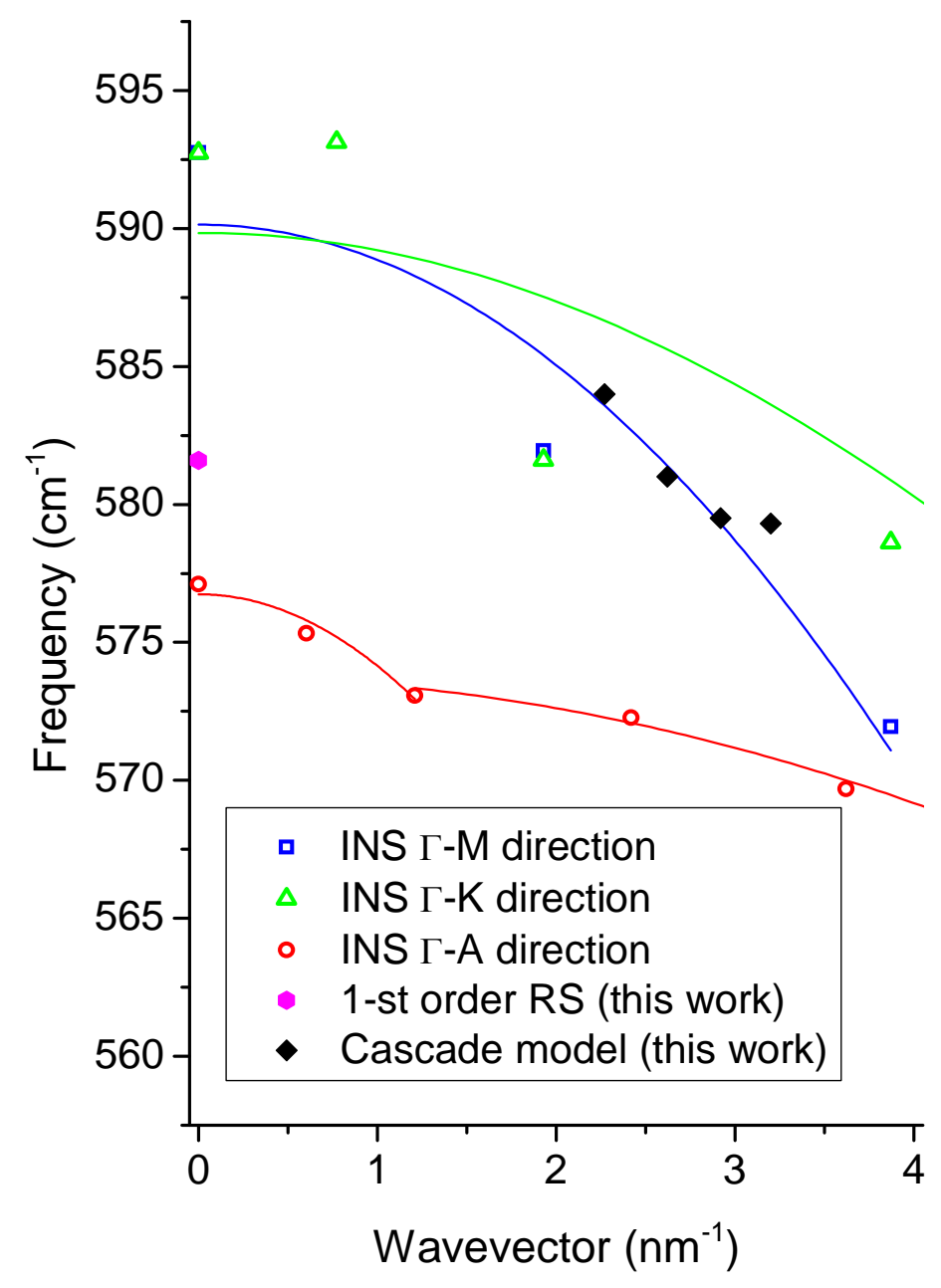

Figure 5 : 